\title{
Some Weighted Norm Estimates for the Composition of the Homotopy and Green's Operator
}

\author{
Huacan $\mathrm{Li}^{1}$ and Qunfang $\mathrm{Li}^{2}$ \\ ${ }^{1}$ School of Science, Jiangxi University of Science and Technology, Ganzhou 341000, China \\ ${ }^{2}$ Department of Mathematics, Ganzhou Teachers College, Ganzhou 341000, China
}

Correspondence should be addressed to Huacan Li; hua03010217@126.com

Received 12 November 2013; Accepted 23 January 2014; Published 2 March 2014

Academic Editor: Yuming Xing

Copyright (c) $2014 \mathrm{H}$. Li and Q. Li. This is an open access article distributed under the Creative Commons Attribution License, which permits unrestricted use, distribution, and reproduction in any medium, provided the original work is properly cited.

\begin{abstract}
We establish the $A_{r}(D)$-weighted integral inequality for the composition of the Homotopy $T$ and Green's operator $G$ on a bounded convex domain and also motivated it to the global domain by the Whitney cover. At the same time, we also obtain some ( $p$, $q)$-type norm inequalities. Finally, as applications of above results, we obtain the upper bound for the $L^{p}$ norms of $T(G(u))$ or $(T(G(u)))_{B}$ in terms of $L^{q}$ norms of $u$ or $d u$.
\end{abstract}

\section{Introduction}

Our purpose is to study the $L^{p}$ theory of the composition of the Homotopy $T$ and Green's operator $G$ acting on differential forms on a bounded convex domain. Both operators play an important role in many fields, including harmonic analysis, potential theory, and partial equations (see [1-6]). In the present paper, we will obtain some $(p, q)$-type norm inequalities for the composition of the Homotopy $T$ and Green's operator $G$ and also prove the $A_{r}(D)$-weighted integral inequality on a bounded convex domain. These results will provide effective tools for studying behavior of solutions of $A$-harmonic equations and related differential systems on manifolds.

We start this paper by introducing some notations and definitions. Let $M$ be a Riemannian, compact, oriented, and $C^{\infty}$-smooth manifold without boundary on $R^{n}$ and let $\Omega$ be an open subset of $R^{n}$. Also, we use $G$ to denote Green's operator throughout this paper. Furthermore, we use $B$ to denote a ball and $\rho B$ to denote the ball with the same center as $B$ and with diameter $(\rho B)=\rho$ diameter $(B)$. We do not distinguish balls from cubs in this paper.

We assume that $\wedge^{k}=\Lambda^{k}\left(R^{n}\right)(k=0,1,2, \ldots, n)$ is the linear space of all $k$-forms $\omega(x)=\sum_{I}(x) d x_{I}=$ $\sum \omega_{i_{1}, i_{2}, \ldots, i_{k}} d x_{i_{1}} \wedge d x_{i_{2}} \wedge \cdots \wedge d x_{i_{k}}$ with summation over all ordered $k$-tuples $I=\left(i_{1}, i_{2}, \ldots, i_{k}\right), 1 \leq i_{1} \leq i_{2} \leq \cdots \leq i_{k} \leq n$. If the coefficient $\omega_{I}(x)$ of $k$-form $\omega(x)$ is differential on $M$, then we call $\omega(x)$ a differential $k$-form on $M$. A differential $k$-form $\omega(x)$ on $M$ is a de Rham current (see [7]) on $M$ with values in $\wedge^{k}\left(R^{n}\right)$. Let $\wedge^{k} M$ be the $k$ th exterior power of the cotangent bundle and $C^{\infty}\left(\wedge^{k} M\right)$ be the space of smooth $k$-forms on $M$. As usual, we use $D^{\prime}\left(M, \wedge^{k}\right)$ to denote the space of all differential $k$-forms and $L^{P}\left(\wedge^{k} M\right)$ to denote the $k$-form $\omega(x)$ with the norm

$$
\begin{aligned}
\|\omega(x)\|_{p, M} & =\left(\int_{M}|\omega(x)|^{p} d x\right)^{1 / p} \\
& =\left(\int_{M}\left(\sum_{I}\left|\omega_{I}(x)\right|^{2}\right)^{p / 2} d x\right)^{1 / p}
\end{aligned}
$$

on $M$. Thus $L^{p}\left(\wedge^{k} M\right)$ is a Banach space. As usual, we still use $\star$ to denote the Hodge star operator. Also, we use $d: D^{\prime}\left(M, \wedge^{k}\right) \rightarrow D^{\prime}\left(M, \wedge^{k+1}\right)$ to denote the differential operator and use $d^{\star}: D^{\prime}\left(M, \wedge^{k+1}\right) \rightarrow D^{\prime}\left(M, \wedge^{k}\right)$ to denote the Hodge codifferential operator which is defined by $d^{\star}=$ $(-1)^{n k+1} \star d \star$ on $D^{\prime}\left(M, \wedge^{k+1}\right)$. The $n$-dimensional Lebesgue measure of a set $E \subseteq R^{n}$ is denoted by $|E|$. We call $w$ a weight if $w \in L_{\text {loc }}^{1}\left(R^{n}\right)$ and $w>0$, a.e. For $0<p<1$, we denote the weighted $L^{p}$-norm of a measurable function $f$ over $M$ by

$$
\|f\|_{p, M, w^{\alpha}}=\left(\int_{M}|f|^{p} w^{\alpha} d x\right)^{1 / p},
$$

where $\alpha$ is a real number. 
Let $D \subset R^{n}$ be a bounded, convex domain. Iwaniec and Lutoborski in [1] first introduced a linear operator $K_{y}$ : $C^{\infty}\left(D, \wedge^{k}\right) \rightarrow C^{\infty}\left(D, \wedge^{k-1}\right)$ satisfying that

$$
\begin{aligned}
&\left(K_{y} \omega\right)\left(x ; \xi_{1}, \xi_{2}, \ldots, \xi_{k-1}\right) \\
& \quad=\int_{0}^{1} t^{k-1} \omega\left(t x+y-t y ; x-y, \xi_{1}, \xi_{2}, \ldots, \xi_{k-1}\right) d t
\end{aligned}
$$

and the decomposition $\omega=d\left(K_{y} \omega\right)+K_{y}(d \omega)$. Then by averaging $K_{y}$ over all points $y$ in $D$, they constructed a Homotopy operator $T: C^{\infty}\left(D, \wedge^{k}\right) \rightarrow C^{\infty}\left(D, \wedge^{k-1}\right)$ satisfying that $T \omega=\int_{D} \varphi(y) K_{y}(\omega) d y$, where $\varphi \in C_{0}^{\infty}(D)$ is normalized by $\int_{D} \varphi(y) d y=1$. The $k$-form $\omega_{D} \in D^{\prime}\left(D, \wedge^{k}\right)$ is defined by $\omega_{D}=(1 /|D|) \int_{D} \omega(y) d y$, if $k=0$, and if $k=1,2, \ldots, n$, then

$$
\begin{gathered}
\omega_{D}=d(T \omega)=\omega-T(d \omega), \\
|T \omega(x)| \leq C \int_{D} \frac{|\omega(y)|}{|y-x|^{n-1}} d y .
\end{gathered}
$$

\section{Boundedness of the \\ Composition of the Homotopy and Green's Operator in $L^{p}$ Space}

In this section, we will prove the $A_{r}(D)$-weighted norm inequality for the composition of the Homotopy $T$ and Green's operator $G$ on a bounded convex domain. Then using the Whitney cover, we develop the local result to the global domain. In [8], Gol'dshtein and Troyanov proved the following lemma.

Lemma 1. Let $D \subset R^{n}$ be a bounded convex domain. The operator $T$ maps $L^{p}\left(D, \wedge^{k}\right)$ continuously to $L^{q}\left(D, \wedge^{k-1}\right)$ in the following cases:

$$
\begin{gathered}
\text { Either } 1 \leq p, q \leq \infty, \quad \frac{1}{p}-\frac{1}{q}<\frac{1}{n}, \\
\text { Or } 1<p, q \leq \infty, \quad \frac{1}{p}-\frac{1}{q} \leq \frac{1}{n} .
\end{gathered}
$$

From [3], we have the following lemma about $L^{s}$ estimates for Green's operator.

Lemma 2. Let $u \in C^{\infty}\left(\wedge^{k} M\right)(k=0,1,2, \ldots, n)$ and $1<s<$ $\infty$. Then there exists a constant $C$, independent of $u$, such that

$$
\begin{gathered}
\left\|d d^{\star} G(u)\right\|_{s, M}+\left\|d^{\star} d G(u)\right\|_{s, M}+\|d G(u)\|_{s, M} \\
+\left\|d^{\star} G(u)\right\|_{s, M}+\|G(u)\|_{s, M} \leq C\|u\|_{s, M} .
\end{gathered}
$$

Definition 3. We say that a weight $w(x)$ satisfies the $A_{r}(D)$ condition for $r>1$ and write $w(x) \in A_{r}(D)$, if $w>0$ a.e. and

$$
\sup _{B \subset D}\left(\frac{1}{|B|} \int_{B} w d x\right)\left(\frac{1}{|B|} \int_{B}\left(\frac{1}{w}\right)^{1 /(r-1)} d x\right)^{r-1}<\infty .
$$

For $A_{r}(D)$ weight, we also need the following result which appears in [9].
Lemma 4. If $w(x) \in A_{r}(D)$, then there exist constants $\beta>1$ and $C$, independent of $w$, such that

$$
\|w\|_{\beta, B} \leq C|B|^{(1-\beta) / \beta}\|w\|_{1, B}
$$

for all balls $B \subset D$.

Theorem 5. Let $D \subset R^{n}$ be a bounded convex domain, $n<p<\infty$, and let $T: L^{p}\left(D, \wedge^{k}\right) \rightarrow L^{p}\left(D, \wedge^{k-1}\right)$ be the Homotopy operator, $k=1,2, \ldots, n$. Then there exists a constant $C$, independent of $u$, such that

$$
\|T(G(u))\|_{p, B, w} \leq C\|u\|_{p, B, w}
$$

for any ball $B \subset D, w(x) \in A_{r}(D)$, and $1<r<p / n$.

Proof. Since $w(x) \in A_{r}(D)$, by Lemma 4, there exist constants $\beta>1$ and $C_{1}$, independent of $w$, such that

$$
\|w\|_{\beta, B} \leq C_{1}|B|^{(1-\beta) / \beta}\|w\|_{1, B}
$$

for any ball $B \subset D$.

Choosing $k=\beta p /(\beta-1)$, then by Hölder inequality with $1 / k+1 / \beta p=1 / p$, we have

$$
\begin{aligned}
\|T(G(u))\|_{p, B, w} & =\left(\int_{B}|T(G(u))|^{p} w(x) d x\right)^{1 / p} \\
& \leq\left(\int_{B}|T(G(u))|^{k} d x\right)^{1 / k}\left(\int_{B} w^{\beta} d x\right)^{1 / \beta p} \\
& =\|T(G(u))\|_{k, B}\|w(x)\|_{\beta, B}^{1 / p} .
\end{aligned}
$$

Thus, substituting (11) into (12), we obtain

$$
\|T(G(u))\|_{p, B, w} \leq C_{1}|B|^{(1-\beta) / \beta p}\|T(G(u))\|_{k, B}\|w(x)\|_{1, B}^{1 / p} .
$$

Taking $m=p / r$, it is easy to see that $m>1$ and $(1 / m)-$ $(1 / k)<(1 / m)<(1 / n)$. Hence communicating Lemmas 1 and 2 , we have

$$
\|T(G(u))\|_{k, B} \leq C_{2}\|G(u)\|_{m, B} \leq C_{3}\|u\|_{m, B} .
$$

Combining (13) and (14), we have

$$
\|T(G(u))\|_{p, B, w} \leq C_{4}|B|^{(1-\beta) / \beta p}\|u\|_{m, B}\|w(x)\|_{1, B}^{1 / p} .
$$

Using Hölder inequality with $1 / p+(r-1) / p=r / p$, we have

$$
\begin{aligned}
\|u\|_{m, B} & \leq\left(\int_{B}\left(|u| w^{1 / p}\right)^{p} d x\right)^{1 / p}\left(\int_{B}\left(\frac{1}{w}\right)^{1 /(r-1)} d x\right)^{(r-1) / p} \\
& =\|u\|_{p, B, w}\left(\int_{B}\left(\frac{1}{w}\right)^{1 /(r-1)} d x\right)^{(r-1) / p} .
\end{aligned}
$$

Note $w(x) \in A_{r}(D)$; then,

$$
\sup _{B \subset D}\left(\frac{1}{|B|} \int_{B} w d x\right)\left(\frac{1}{|B|} \int_{B}\left(\frac{1}{w}\right)^{1 /(r-1)} d x\right)^{r-1}<C_{5}<\infty .
$$


Thus, observing (15) and (16), we immediately obtain that

$$
\begin{aligned}
\|T(G(u))\|_{p, B, w} & \leq C_{6}|B|^{(1-\beta) / \beta p+(r / p)}\|u\|_{p, B, w} \\
& \leq C_{6}|D|^{(1-\beta) / \beta p+(r / p)}\|u\|_{p, B, w} \leq C_{7}\|u\|_{p, B, w} .
\end{aligned}
$$

Here $C_{7}$ is a constant independent of $u$. Thus we complete the proof of Theorem 5 .

Furthermore, if $u$ is an $A$-harmonic tensor on $D, \rho>1$ and $0<s, t<\infty$, then there exists a constant $C$, independent of $u$, such that

$$
\|u\|_{s, B} \leq C|B|^{(t-s) / t s}\|u\|_{t, \rho B}
$$

for all balls or cubs $B$ with $\rho B \subset D$ (for more details about $A$ harmonic tensors, see [10]). By the property of $A$-harmonic tensor, using the same method developed in the proof of Theorem 5, we can easily extend into the following $A_{r}(D)$ weighted version.

Corollary 6. Let $D \subset R^{n}$ be a bounded convex domain, $n<$ $p<\infty, u$ be an A-harmonic tensor, and $T: L^{p}\left(D, \wedge^{k}\right) \rightarrow$ $L^{p}\left(D, \wedge^{k-1}\right)$ be the Homotopy operator, $k=1,2, \ldots, n$. Then there exists a constant $C$, independent of $u$, such that

$$
\|T(G(u))\|_{p, B, w^{\alpha}} \leq C\|u\|_{p, \rho B, w^{\alpha}}
$$

for any ball $B \subset D, w(x) \in A_{r}(D)$, and $1<r<p / n, 0<\alpha \leq$ $1, \rho>1$.

In order to obtain the boundedness of the composition $T \circ G$, we need the following modified Whitney cover in [10] and see [11] for more details about Whitney cover.

Lemma 7. Each open subset $E \subset R^{n}$ has a modified Whitney cover of cubs $W=\left\{Q_{i}\right\}$ satisfying $\bigcup_{i} Q_{i}=E$ and $\sum_{\mathrm{Q}_{i} \in W} \chi_{\sqrt{5 / 4} \mathrm{Q}_{i}} \leq N \cdot \chi_{E}(x)$, for all $x \in R^{n}$ and some $N>1$, where $\chi_{E}(x)$ is the characteristic function for the set $E$.

Theorem 8. Let $D \subset R^{n}$ be a bounded convex domain, $n<$ $p<\infty$. Then the composite operator $T \circ G: L^{p}\left(D, \wedge^{k}, w\right) \rightarrow$ $L^{p}\left(D, \wedge^{k-1}, w\right)$ is bounded, $k=1,2, \ldots, n$. Here $w(x) \in A_{r}(D)$ and $1<r<p / n$.

Proof. From Lemma 7, we know that there exists a sequence of cubs $W=\left\{Q_{i}\right\}$ such that $\bigcup_{i} Q_{i}=D$ and $\sum_{Q_{i} \in W} \chi_{\sqrt{5 / 4} Q_{i}} \leq$ $N \cdot \chi_{E}(x)$ for all $x \in D$, where $N>1$ is some constant. Hence, for $u \in L^{p}\left(D, \wedge^{k}, w\right)$, we have

$$
\begin{aligned}
\| T & (G(u)) \|_{p, D, w}^{p} \\
& =\int_{D}|T(G(u))|^{p} d \mu \leq \sum_{Q_{i} \in W} \int_{Q_{i}}|T(G(u))|^{p} d \mu \\
& \leq \sum_{Q_{i} \in W} C_{1} \int_{Q_{i}}|u|^{p} d \mu \leq \sum_{Q_{i} \in W} C_{1} \int_{D}|u|^{p} \chi_{Q_{i}}(x) d \mu
\end{aligned}
$$

$$
\begin{aligned}
& \leq C_{1} \int_{D} \sum_{Q_{i} \in W}|u|^{p} \chi_{Q_{i}}(x) d \mu \leq C_{1} \int_{D} N \cdot|u|^{p} \chi_{D}(x) d \mu \\
& \leq C_{1} N \int_{D}|u|^{p} d \mu=C_{2} \int_{D}|u|^{p} d \mu=C_{2}\|u\|_{p, D, w}^{p}
\end{aligned}
$$

where $d \mu=w(x) d x$ and $C_{2}=C_{1} N$ is independent of $u$ and each $Q_{i}$. Thus, we complete the proof of Theorem 8 .

\section{Norm Estimates with Power-Type Weights}

Let $S \subset R^{n}$ be a bounded domain and $D$ be a nonempty of $\bar{S}=S \bigcup \partial S$. If we use $\operatorname{dist}(x, D)$ to denote the distance of the point $x$ from the set $D$, then $\omega(x)=(\operatorname{dist}(x, D))^{\varepsilon}$ for $\varepsilon \in R$ is called power-type weight. In this section, we will establish some strong $(p, q)$-type norm inequalities with power-type weights for the composition of the Homotopy $T$ and Green's operator $G$ acting on differential form. In the following proof, we will use the following Lemma which appears in [8].

Lemma 9. The operator $T: \Omega_{p, r}\left(D, \wedge^{k}\right) \rightarrow \Omega_{q, p}\left(D, \wedge^{k-1}\right)$ is bounded provided that

$$
\begin{gathered}
\text { Either } 1 \leq p, q, r \leq \infty, \quad \frac{1}{p}-\frac{1}{q}<\frac{1}{n}, \quad \frac{1}{r}-\frac{1}{p}<\frac{1}{n}, \\
\text { Or } 1<p, q, r \leq \infty, \quad \frac{1}{p}-\frac{1}{q} \leq \frac{1}{n}, \quad \frac{1}{r}-\frac{1}{p} \leq \frac{1}{n} .
\end{gathered}
$$

Theorem 10. Let $D \subset R^{n}$ be a bounded convex domain, $1<p$, $q<\infty, 0 \leq 1 / p-1 / q \leq 1 / n$, and let $T: L^{p}\left(D, \wedge^{k}\right) \rightarrow$ $L^{q}\left(D, \wedge^{k-1}\right)$ be the Homotopy operator, $k=1,2, \ldots, n$. Then there exists a constant $C$, independent of $u$, such that

$$
\left\|T(G(u))-(T(G(u)))_{D}\right\|_{q, D} \leq C(1+\operatorname{diam}(D))\|u\|_{p, D}
$$

for any $u \in \Omega_{p, p}\left(D, \wedge^{k}\right)$

Proof. From (4), we have the following decomposition:

$$
G(u)=T(d(G(u)))+d(T(G(u)))
$$

for any differential form $u \in \Omega_{p, p}\left(D, \wedge^{k}\right), k=1,2, \ldots, n$.

Note that $u$ is an element of $\Omega_{p, p}\left(D, \wedge^{k}\right), k=1,2, \ldots, n$. From (4) and Lemmas 1 and 9, we have

$$
\begin{array}{r}
\left\|T(G(u))-(T(G(u)))_{D}\right\|_{q, D} \\
\quad=\|T(d(T(G(u))))\|_{q, D} \\
\leq C_{1}\|d(T(G(u)))\|_{p, D} .
\end{array}
$$

Here $C_{1}$ is a constant independent of $u$. Applying (24) and (5), we have

$$
\begin{aligned}
\| d & (T(G(u))) \|_{p, D} \\
& =\|G(u)-T(d(G(u)))\|_{p, D} \\
& \leq\|G(u)\|_{p, D}+\|T(d(G(u)))\|_{p, D} \\
& \leq\|G(u)\|_{p, D}+C_{2} \operatorname{diam}(D)\|d(G(u))\|_{p, D} .
\end{aligned}
$$


Applying Lemma 2 into (26), we obtain

$$
\|d(T(G(u)))\|_{p, D} \leq\left(C_{3}+C_{4} \operatorname{diam}(D)\right)\|u\|_{p, D} .
$$

Thus

$$
\begin{aligned}
& \left\|T(G(u))-(T(G(u)))_{D}\right\|_{q, D} \\
& \quad \leq\left(C_{5}+C_{6} \operatorname{diam}(D)\right)\|u\|_{p, D} \\
& \leq C_{7}(1+\operatorname{diam}(D))\|u\|_{p, D} .
\end{aligned}
$$

Here $C_{7}=\max \left\{C_{5}, C_{6}\right\}$ is independent of $u$. Thus, we complete the proof of Theorem 10 .

Next, we consider the following norm comparison equipped with power-type weights.

Theorem 11. Let $D \subset R^{n}$ be a bounded convex domain, $1<p$, $q<\infty, 0 \leq 1 / p-1 / q \leq 1 / n$, let $T: L^{p}\left(D, \wedge^{k}\right) \rightarrow$ $L^{q}\left(D, \wedge^{k-1}\right)$ be the Homotopy operator, $k=1,2, \ldots, n$, and that continuous functions $h$ and $g$ defined in $(0,+\infty)$ satisfy (1) $\lim _{t \rightarrow 0} h(t)=0 ;(2) \lim _{t \rightarrow 0} g(t)=\infty$. Then there exists $a$ constant $C$, independent of $u$, such that

$$
\left\|T(G(u))-(T(G(u)))_{D}\right\|_{q, D, \mu_{1}} \leq C(1+\operatorname{diam}(D))\|u\|_{p, D, \mu_{2}}
$$

for any $u \in \Omega_{p, p}\left(D, \wedge^{k}\right), d \mu_{1}=h(\operatorname{dist}(x, \partial D)) d x, d \mu_{2}=$ $g(\operatorname{dist}(x, \partial D)) d x$.

Proof. From Theorem 10, we know that there exists a constant $C_{1}$, independent of $u$, such that

$$
\left\|T(G(u))-(T(G(u)))_{D}\right\|_{q, D} \leq C_{1}(1+\operatorname{diam}(D))\|u\|_{p, D} .
$$

Fixing $\varepsilon>0$, then there exists $\delta_{1}(\varepsilon)>0$ such that $h(\operatorname{dist}(x, \partial D))<\varepsilon$ for all $x \in D$ with $\operatorname{dist}(x, \partial D)<\delta_{1}$. Let $D_{1}=\left\{x \in D, \operatorname{dist}(x, \partial D)<\delta_{1}\right\}$ and $D_{2}=D-D_{1}$. Then for all $x \in D_{2}$, we have

$$
\delta_{1} \leq \operatorname{dist}(x, \partial D)<\operatorname{diam}(D) .
$$

Therefore, by the continuity of $h$, we know that there exists $M_{1}>0$, such that

$$
h(\operatorname{dist}(x, \partial D))<M_{1}
$$

for all $x \in D_{2}$. Thus we have

$$
\begin{aligned}
& \left\|T(G(u))-(T(G(u)))_{D}\right\|_{q, D, \mu_{1}} \\
& =\left(\int_{D}\left|T(G(u))-(T(G(u)))_{D}\right|^{q} \cdot h(\operatorname{dist}(x, \partial D)) d x\right)^{1 / q} \\
& \leq\left(\varepsilon \int_{D_{1}}\left|T(G(u))-(T(G(u)))_{D}\right|^{q} d x\right. \\
& \left.\quad+M_{1} \int_{D_{2}}\left|T(G(u))-(T(G(u)))_{D}\right|^{q} d x\right)^{1 / q} \\
& \leq C_{2}\left(\int_{D}\left|T(G(u))-(T(G(u)))_{D}\right|^{q} d x\right)^{1 / q} .
\end{aligned}
$$

Here $C_{2}=\max \left\{\varepsilon^{1 / q}, M_{1}^{1 / q}\right\}$. Communicating (30) and (33), we have

$$
\begin{aligned}
& \left\|T(G(u))-(T(G(u)))_{D}\right\|_{q, D, \mu_{1}} \\
& \quad \leq C_{2}\left\|T(G(u))-(T(G(u)))_{D}\right\|_{q, D} \\
& \quad \leq C_{3}(1+\operatorname{diam}(D))\|u\|_{p, D} .
\end{aligned}
$$

Note that $\lim _{t \rightarrow 0}(1 / g(t))=0$. Then there exists $\delta_{2}(\varepsilon)>0$ such that $1 / g(\operatorname{dist}(x, \partial D))<\varepsilon$ for all $x \in D$ with $\operatorname{dist}(x, \partial D)<$ $\delta_{2}$. Let $D_{1}^{\prime}=\left\{x \in D, \operatorname{dist}(x, \partial D)<\delta_{2}\right\}$ and $D_{2}^{\prime}=D-D_{1}^{\prime}$. Then for all $x \in D_{2}^{\prime}$, we have

$$
\delta_{2} \leq \operatorname{dist}(x, \partial D)<\operatorname{diam}(D) .
$$

Therefore, by the continuity of $g$, we know that there exists $M_{2}>0$, such that

$$
\frac{1}{g(\operatorname{dist}(x, \partial D))}<M_{2}
$$

for all $x \in D_{2}^{\prime}$. Therefore, we obtain

$$
\begin{aligned}
\|u\|_{p, D} & =\left(\int_{D}|u|^{p} \frac{1}{g(\operatorname{dist}(x, \partial D))} d \mu_{2}\right)^{1 / p} \\
& \leq\left(\varepsilon \int_{D_{1}^{\prime}}|u|^{p} d \mu_{2}+M_{2} \int_{D_{2}^{\prime}}|u|^{p} d \mu_{2}\right)^{1 / p} \\
& \leq C_{4}\left(\int_{D}|u|^{p} d \mu_{2}\right)^{1 / p}=C_{4}\|u\|_{p, D, \mu_{2}} .
\end{aligned}
$$

Here $C_{4}=\max \left\{\varepsilon^{1 / p}, M_{2}^{1 / p}\right\}$. By (34) and (37), we have

$$
\begin{aligned}
& \left\|T(G(u))-(T(G(u)))_{D}\right\|_{q, D, \mu_{1}} \\
& \quad \leq C_{5}(1+\operatorname{diam}(D))\|u\|_{p, D, \mu_{2}} .
\end{aligned}
$$

Here $C_{5}$ is independent of $u$. Thus, we complete the proof of Theorem 11.

In Theorem 11, if we choose $h(t)=t^{r}$ and $g(t)=t^{-s}, 0<r$, $s<\infty$, we can easily obtain the following corollary.

Corollary 12. Let $D \subset R^{n}$ be a bounded convex domain, $1<$ $p, q<\infty, 0 \leq 1 / p-1 / q \leq 1 / n$, and let $T: L^{p}\left(D, \wedge^{k}\right) \rightarrow$ $L^{q}\left(D, \wedge^{k-1}\right)$ be the Homotopy operator, $k=1,2, \ldots, n$. Then there exists a constant $C$, independent of $u$, such that

$$
\begin{aligned}
\int_{D} \mid T & (G(u))-\left.(T(G(u)))_{D}\right|^{q} \cdot(\operatorname{dist}(x, \partial D))^{r} d x \\
& \leq C(1+\operatorname{diam}(D))\left(\int_{D}|u|^{p} \frac{1}{(\operatorname{dist}(x, \partial D))^{s}} d x\right)^{1 / p} .
\end{aligned}
$$

Here $0<r, s<\infty$.

Note that, in the proof of Theorem 11, if we let the composite operator $T \circ G$ act on the solution of nonhomogeneous $A$ harmonic equation, then we can $\operatorname{drop} \lim _{t \rightarrow 0} h(t)=0$. Next, we state the result as follows. 
Corollary 13. Let $D \subset R^{n}$ be a bounded convex domain, $1<p, q<\infty, 0 \leq 1 / p-1 / q \leq 1 / n$, let $T: L^{p}\left(D, \wedge^{k}\right) \rightarrow$ $L^{q}\left(D, \wedge^{k-1}\right)$ be the Homotopy operator, and $u \in \Omega_{p, p}\left(D, \wedge^{k}\right)$ is a solution of nonhomogeneous $A$-harmonic equation, $k=$ $1,2, \ldots, n$. If continuous functions $h$ and $g$ defined in $(0,+\infty)$ satisfy that $\lim _{t \rightarrow 0} g(t)=\infty, d \mu_{1}=h(\operatorname{dist}(x, \partial D)) d x$ and $d \mu_{2}=g(\operatorname{dist}(x, \partial D)) d x$. Then there exists a constant $C$, independent of $u$, such that

$$
\left\|T(G(u))-(T(G(u)))_{D}\right\|_{q, B, \mu_{1}} \leq C(1+\operatorname{diam}(D))\|u\|_{p, \rho B, \mu_{2}}
$$

for all balls $B$ with $\rho B \subset D$. Here $\rho>1$ is some constant.

It is easy to find that the above corollary does not hold for balls $B \subset D$ with $\partial B \bigcap \partial D \neq \Phi$ but holds for those balls with $\rho B \subset D$. Next, we introduce the following singular integral inequality.

Theorem 14. Let $D \subset R^{n}$ be a bounded convex domain, $1<p$, $q<\infty, 0 \leq 1 / p-1 / q \leq 1 / n, \operatorname{let} T: L^{p}\left(D, \wedge^{k}\right) \rightarrow L^{q}\left(D, \wedge^{k-1}\right)$ be the Homotopy operator, and $u \in \Omega_{p, p}\left(D, \wedge^{k}\right)$ is a solution of nonhomogeneous A-harmonic equation, $k=1,2, \ldots, n$. If continuous functions $h$ and $g$ defined in $(0,+\infty)$ and $h(t)$ is an increasing function, then there exists a constant $C$, independent of $u$, such that

$$
\begin{aligned}
& \left(\int_{B}\left|T(G(u))-(T(G(u)))_{B}\right|^{q} \frac{1}{g(\operatorname{dist}(x, \partial D))} d x\right)^{1 / q} \\
& \leq C \\
& \quad \times\left(\int_{\rho B} \frac{|u|^{p}}{(h(\operatorname{dist}(x, \partial D)))^{\lambda}} d x\right)^{1 / p}
\end{aligned}
$$

for all balls $B$ with $\rho B \subset D$ and $0<\lambda<1$. Here $\rho>1$ is some constant.

Proof. Let $k=q /(1-\lambda)$. From $0<\lambda<1$, it is easy to see that $k>q$. Using the Hölder inequality, we have

$$
\begin{aligned}
& \left(\int_{B}\left|T(G(u))-(T(G(u)))_{B}\right|^{q} \frac{1}{g(\operatorname{dist}(x, \partial D))} d x\right)^{1 / q} \\
& \leq\left(\int_{B}\left|T(G(u))-(T(G(u)))_{B}\right|^{k} d x\right)^{1 / k} \\
& \quad \times\left(\int_{B} \frac{1}{(g(\operatorname{dist}(x, \partial D)))^{k /(k-q)}} d x\right)^{(k-q) / k q} \\
& =\left\|T(G(u))-(T(G(u)))_{B}\right\|_{k, B} \\
& \quad \times\left(\int_{B} \frac{1}{(g(\operatorname{dist}(x, \partial D)))^{k /(k-q)}} d x\right)^{(k-q) / k q} .
\end{aligned}
$$

Note that $\rho B \subset D$. Therefore, there exists a positive number $c$ such that

$$
c<\operatorname{dist}(x, \partial D) \leq \operatorname{diam}(D)
$$

for all $x \in B$. Furthermore, by the continuity of function $g$ in $(0,+\infty), g(\operatorname{dist}(x, \partial D))$ has a positive lower bound $M$ in $B$. Thus, from Theorem 10 and (42), we have

$$
\begin{aligned}
\left(\int_{B} \mid\right. & \left.T(G(u))-\left.(T(G(u)))_{B}\right|^{q} \frac{1}{g(\operatorname{dist}(x, \partial D))} d x\right)^{1 / q} \\
\leq & \left(\frac{1}{M}\right)^{1 / q}|B|^{(k-q) / k q}\left\|T(G(u))-(T(G(u)))_{B}\right\|_{k, B} \\
\leq & C_{1}|B|^{(k-q) / k q}(1+\operatorname{diam}(B))\|u\|_{k, B} \\
& \leq C_{1}|B|^{(k-q) / k q}(1+\operatorname{diam}(B))\|u\|_{k, \rho_{1} B},
\end{aligned}
$$

where $\rho_{1}>1$ is a constant. Let $\varepsilon \in(1 / p, 1)$ and $m=\varepsilon p$. Since $u$ is the solution of nonhomogenous $A$-harmonic equation. By (19), we know

$$
\|u\|_{k, \rho_{1} B} \leq C_{2}\left|\rho_{1} B\right|^{(m-k) / m k}\|u\|_{m, \rho B},
$$

where $\rho>\rho_{1}>1$ is a constant. It is easy to find that $1<m<$ p. Using the Hölder inequality, we have

$$
\begin{aligned}
\|u\|_{m, \rho B}= & \left(\int_{\rho B}|u|^{m} \frac{1}{(h(\operatorname{dist}(x, \partial D)))^{m \lambda / p}}\right. \\
& \left.\cdot(h(\operatorname{dist}(x, \partial D)))^{m \lambda / p} d x\right)^{1 / m} \\
\leq & \left(\int_{\rho B} \frac{|u|^{p}}{(h(\operatorname{dist}(x, \partial D)))^{\lambda}} d x\right)^{1 / p} \\
& \times\left(\int_{\rho B}\left((h(\operatorname{dist}(x, \partial D)))^{\lambda / p}\right)^{m p /(p-m)} d x\right)^{(p-m) / m p} .
\end{aligned}
$$

The continuity and monotonicity of function $h$ imply that

$$
\begin{aligned}
& \left(\int_{\rho B}\left((h(\operatorname{dist}(x, \partial D)))^{\lambda / p}\right)^{m p /(p-m)} d x\right)^{(p-m) / m p} \\
& \quad=\left(\int_{\rho B}(h(\operatorname{dist}(x, \partial D)))^{\varepsilon \lambda /(1-\varepsilon)} d x\right)^{(1-\varepsilon) / \varepsilon p} \\
& \quad \leq|\rho B|^{(1-\varepsilon) / \varepsilon p}(h(\operatorname{diam}(D)))^{\lambda / p} .
\end{aligned}
$$

Hence, combining (41)-(47), we have

$$
\begin{aligned}
\left(\int_{B}\left|T(G(u))-(T(G(u)))_{B}\right|^{q} \frac{1}{g(\operatorname{dist}(x, \partial D))} d x\right)^{1 / q} \\
\leq C_{3}|B|^{(k-q) / k q}(1+\operatorname{diam}(B))\left|\rho_{1} B\right|^{(m-k) / m k}|\rho B|^{(1-\varepsilon) / \varepsilon p} \\
\quad \times(h(\operatorname{diam}(D)))^{\lambda / p}\left(\int_{\rho B} \frac{|u|^{p}}{(h(\operatorname{dist}(x, \partial D)))^{\lambda}} d x\right)^{1 / p} \\
\leq C_{4}(1+\operatorname{diam}(B))|\rho B|^{(p-q) / p q} \\
\quad \times\left(\int_{\rho B} \frac{|u|^{p}}{(h(\operatorname{dist}(x, \partial D)))^{\lambda}} d x\right)^{1 / p} .
\end{aligned}
$$

Here $C_{4}$ is dependent of $B$ and $h$ but independent of $u$. Thus, we complete the proof of Theorem 11. 


\section{Application}

In this section, we will use the estimates in Section 3 to obtain the upper bound for the $L^{p}$ norms of $T(G(u))$ or $(T(G(u)))_{B}$ in terms of $L^{q}$ norms of $u$ or $d u$.

Example 15. For $n \geq 2$, let $u$ be a $(n-1)$-form defined in $R^{n}$ by

$$
\begin{aligned}
u= & \frac{x_{1}}{\sqrt{x_{1}^{2}+x_{2}^{2}+\cdots+x_{n}^{2}}} d x_{2} \wedge d x_{3} \wedge \cdots \wedge d x_{n} \\
& -\frac{x_{2}}{\sqrt{x_{1}^{2}+x_{2}^{2}+\cdots+x_{n}^{2}}} d x_{1} \wedge d x_{3} \wedge \cdots \wedge d x_{n} \\
& +\cdots+(-1)^{n-1} \\
& \times \frac{x_{n}}{\sqrt{x_{1}^{2}+x_{2}^{2}+\cdots+x_{n}^{2}}} d x_{1} \wedge d x_{2} \wedge \cdots \wedge d x_{n-1} .
\end{aligned}
$$

It is easy to find that

$$
|u|=1, \quad d u=\frac{n-1}{\sqrt{x_{1}^{2}+x_{2}^{2}+\cdots+x_{n}^{2}}} d x_{1} \wedge d x_{2} \wedge \cdots \wedge d x_{n} .
$$

If we choose the usual $(p, p)$-type norm inequality to estimate $T(G(u))-(T(G(u)))_{B}$ and take $p=n$, where $B=B(O, r) \subset R^{n}$ is a ball, then by Theorem 10, we have

$$
\begin{gathered}
\left(\int_{B}\left|T(G(u))-(T(G(u)))_{B}\right|^{n} d x\right)^{1 / n} \\
\leq C_{1}(1+\operatorname{diam}(B))\left(\int_{B}|u|^{n} d x\right)^{1 / n} \\
\quad=C_{1}(1+\operatorname{diam}(B))|B|^{1 / n} .
\end{gathered}
$$

However, if we choose the $(p, q)$-type norm inequality to estimate $T(G(u))-(T(G(u)))_{B}$ and take $p=n-1, q=n$, then $p, q$ satisfy the condition $0 \leq 1 / p-1 / q \leq 1 / n$. Hence by using Theorem 10, we obtain

$$
\begin{aligned}
\left(\int_{B} \mid\right. & \left.T(G(u))-\left.(T(G(u)))_{B}\right|^{n} d x\right)^{1 / n} \\
& \leq C_{2}(1+\operatorname{diam}(B))\left(\int_{B}|u|^{n-1} d x\right)^{1 /(n-1)} \\
& =C_{2}(1+\operatorname{diam}(B))|B|^{1 /(n-1)} .
\end{aligned}
$$

Compare (51) and (52), we can easily find that if we choose different $(p, q)$-type norm inequality to estimate the oscillation $T(G(u))-(T(G(u)))_{B}$, we also obtain the different upper bound.

Example 16. In $R^{2}$, consider that

$$
u(x, y)=\arctan \frac{y}{x-1}-\arctan \frac{y}{x+1} .
$$

It is easy to check that $u(x, y)$ is harmonic in the upper half plane. Note that

$$
\begin{gathered}
d u=\frac{\partial u}{\partial x} d x+\frac{\partial u}{\partial y} d y, \\
* d u=\frac{\partial u}{\partial x} d y-\frac{\partial u}{\partial y} d x .
\end{gathered}
$$

Therefore, we have

$$
d * d u=\left(\frac{\partial^{2} u}{\partial x^{2}}+\frac{\partial^{2} u}{\partial y^{2}}\right) d x \wedge d y=0
$$

which implies that $* d u$ is a closed form and hence is a solution of nonhomogenous $A$-harmonic equation. It is easy to see that

$$
|* d u|=\frac{1}{\sqrt{\left((x-1)^{2}+y^{2}\right)\left((x+1)^{2}+y^{2}\right)}} .
$$

Let $D$ denote a bound convex domain in the upper half plane and let $\sigma \bar{B} \subset D$ be a closed ball without the points $(-1,0)$ and $(1,0)$. If $\sigma \bar{B}$ and $D$ satisfy that $\operatorname{dist}(\sigma B, \partial D)=M>0$, then both $|* d u|$ and $(\operatorname{dist}(x, \partial D))^{-1}$ have the upper bounds in $\sigma \bar{B}$. Thus, for the term

$$
\int_{B}\left|T(G(u))-(T(G(u)))_{B}\right|^{p} \frac{1}{g(\operatorname{dist}(x, \partial D))} d x,
$$

it is usually not easy to be estimated due to the complexity of the compositions $T(G(u))$ and the function $g$. However, by Theorem 14, (57) can be controlled by the term

$$
\int_{\rho B} \frac{|u|^{p}}{(h(\operatorname{dist}(x, \partial D)))^{\lambda}} d x
$$

Thus, we obtain an upper bound of (57).

\section{Conflict of Interests}

The authors declare that there is no conflict of interests regarding the publication of this paper.

\section{Acknowledgments}

The first author was supported by the foundation at the Jiangxi University of Science and Technology (no. jxxj12073) and by the Youth Foundation of Jiangxi Provincial Education Department of China (no. GJJ13376).

\section{References}

[1] T. Iwaniec and A. Lutoborski, "Integral estimates for null Lagrangians," Archive for Rational Mechanics and Analysis, vol. 125, no. 1, pp. 25-79, 1993.

[2] S. Ding and B. Liu, "A singular integral of the composite operator," Applied Mathematics Letters, vol. 22, no. 8, pp. 1271-1275, 2009. 
[3] C. Scott, " $L^{p}$ theory of differential forms on manifolds," Transactions of the American Mathematical Society, vol. 347, no. 6, pp. 2075-2096, 1995.

[4] S. Ding, "Integral estimates for the Laplace-Beltrami and Green's operators applied to differential forms on manifolds," Zeitschrift Für Analysis und Ihre Anwendungen, vol. 22, no. 4, pp. 939-957, 2003.

[5] H. Bi and S. Ding, "Some strong $(p, q)$-type inequalities for the homotopy operator," Computers \& Mathematics with Applications, vol. 62, no. 4, pp. 1780-1789, 2011.

[6] Y. Xing and S. Ding, "Poincaré inequalities with the Radon measure for differential forms," Computers \& Mathematics with Applications, vol. 59, no. 6, pp. 1944-1952, 2010.

[7] G. de Rham, Differential Manifolds, Springer, Berlin, Germany, 1980.

[8] V. Gol'dshtein and M. Troyanov, "Sobolev inequalities for differential forms and $L_{q, p}$-cohomology," The Journal of Geometric Analysis, vol. 16, no. 4, pp. 597-631, 2006.

[9] J. B. Garnett, Bounded Analytic Functions, Academic Press, New York, NY, USA, 1970.

[10] C. A. Nolder, "Hardy-Littlewood theorems for A-harmonic tensors," Illinois Journal of Mathematics, vol. 43, no. 4, pp. 613-631, 1999.

[11] E. M. Stein, Singular Integrals and Differentiability Properties of Functions, Princeton University Press, Princeton, NJ, USA, 1970. 


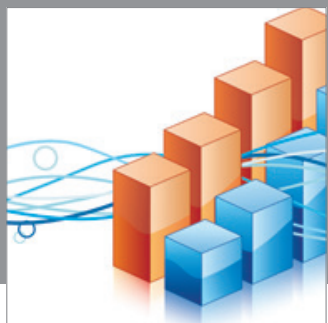

Advances in

Operations Research

mansans

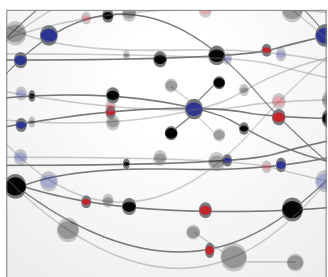

The Scientific World Journal
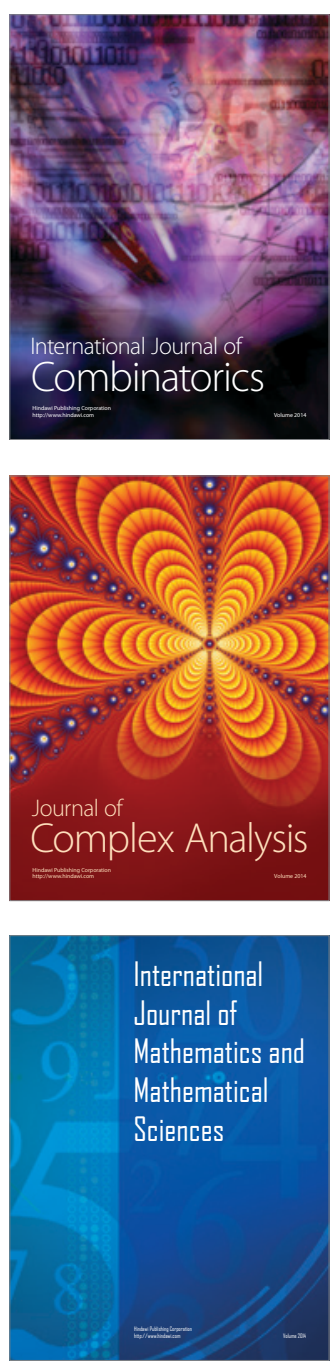
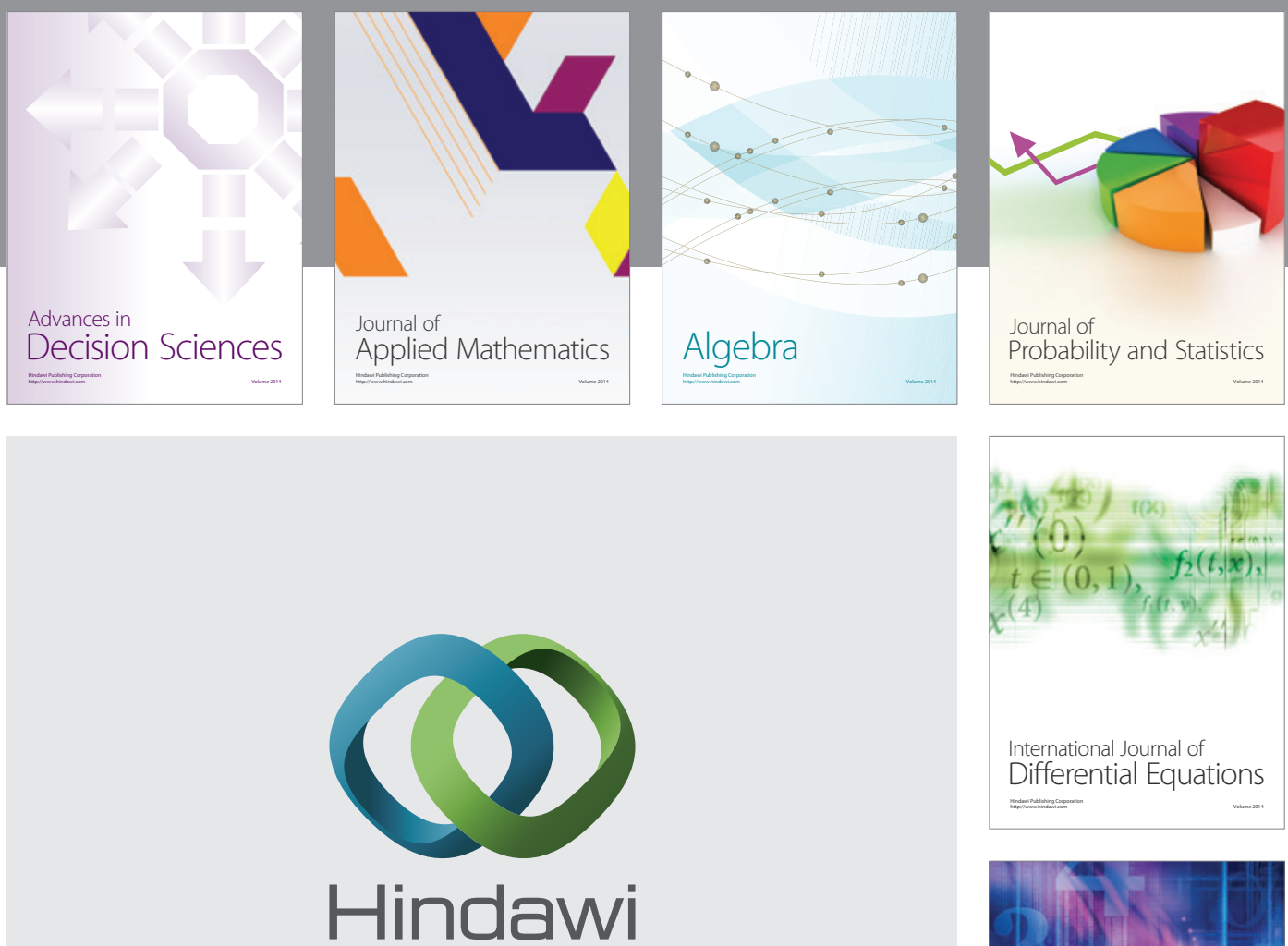

Submit your manuscripts at http://www.hindawi.com
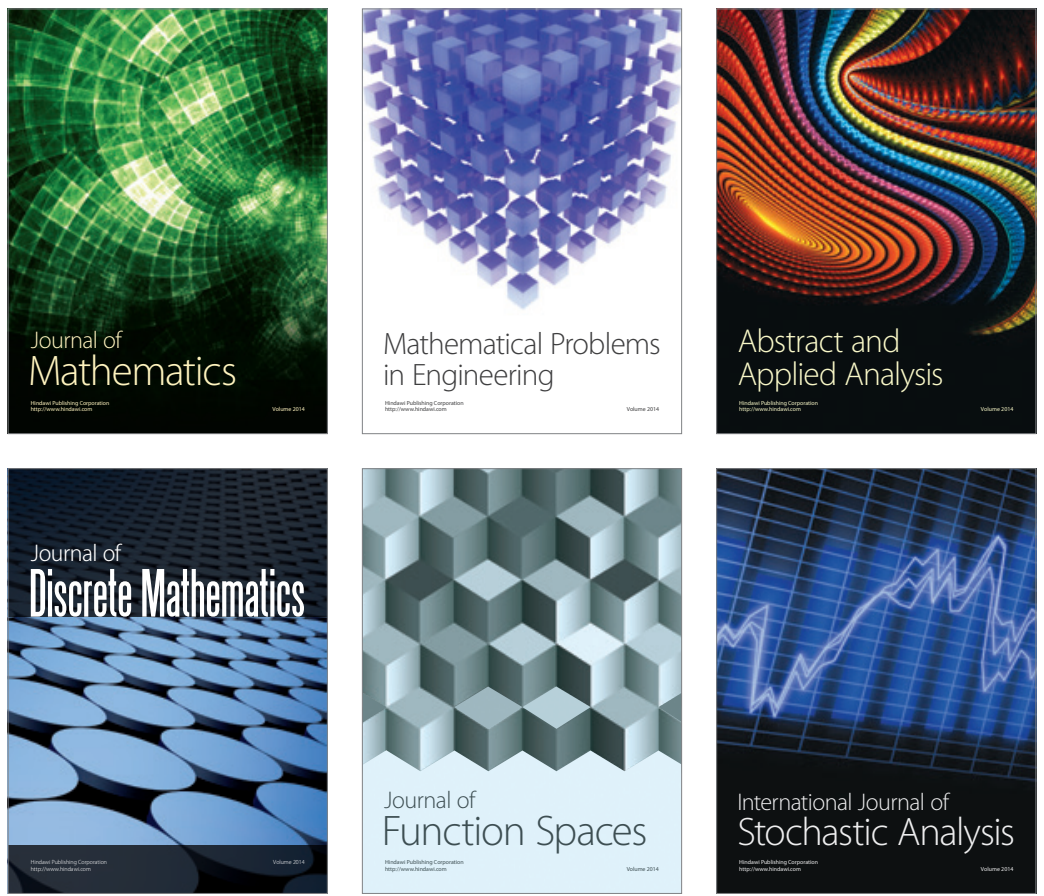

Journal of

Function Spaces

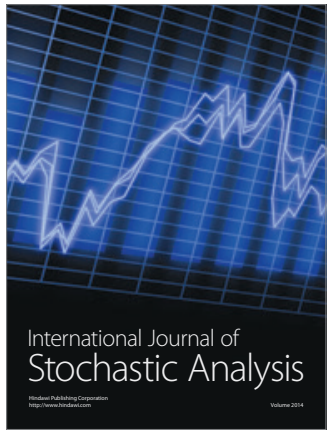

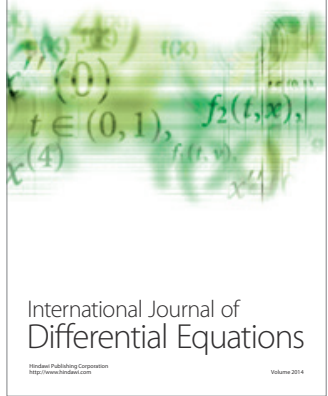
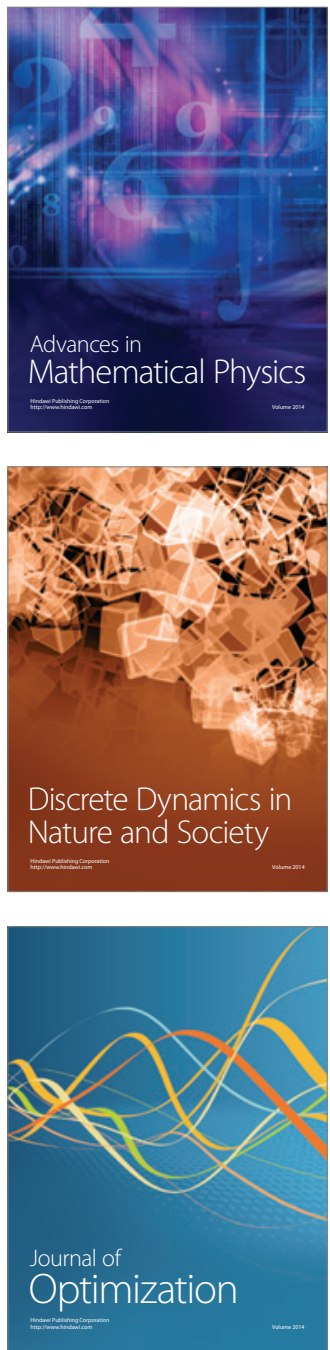